\title{
Trophoblast Cell-surface Antigen 2 Expression in Advanced Lung Cancer Patients and the Effects of Anti-cancer Treatments
}

Shota Omori ( $\nabla$ s.omori@scchr.jp )

Shizuoka Cancer Center https://orcid.org/0000-0003-1407-9779

Koji Muramatsu

Shizuoka Cancer Center

Takuya Kawata

Shizuoka Cancer Center

Eriko Miyawaki

Shizuoka Cancer Center: Shizuoka Kenritsu Shizuoka Gan Center

Taichi Miyawaki

Shizuoka Cancer Center

Nobuaki Mamesaya

Shizuoka Cancer Center

Takahisa Kawamura

Osaka International Cancer Institute

Haruki Kobayashi

Shizuoka Cancer Center

Kazuhisa Nakashima

Shimane University Faculty of Medicine

Kazushige Wakuda

Shizuoka Cancer Center

Akira Ono

Shizuoka Cancer Center

Hirotsugu Kenmotsu

Shizuoka Cancer Center

Tateaki Naito

Shizuoka Cancer Center

Haruyasu Murakami

Shizuoka Cancer Center

Takashi Sugino

Shizuoka Cancer Center

Toshiaki Takahashi 


\section{Research Article}

Keywords: Trophoblast cell-surface antigen 2, immunohistochemical analysis, chemotherapy, targeted therapy, thoracic radiotherapy

Posted Date: March 10th, 2021

DOI: https://doi.org/10.21203/rs.3.rs-270630/v1

License: (c) (i) This work is licensed under a Creative Commons Attribution 4.0 International License. Read Full License 


\section{Abstract}

Background: Trophoblast cell-surface antigen 2 (TROP2) is expressed on the surface of trophoblast cells and many malignant tumor cells. However, data on TROP2 expression in advanced lung cancer is insufficient, and its changes have not been fully evaluated.

Methods: We assessed the prevalence and changes in TROP2 expression in lung cancer patients receiving anti-cancer treatments using immunohistochemical $(\mathrm{IHC}$ ) analysis with an anti-TROP2 (clone: SP295). IHC scores were graded from $0-3$; grade $\geq 2$ was considered positive for TROP2 expression. We defined a difference in IHC score, before and after anti-cancer treatments, as the change in TROP2 expression.

Results: Before anti-cancer treatment, TROP2 expression was observed in 89\% (143/160) of patients and was significantly more common in adenocarcinoma and squamous cell carcinoma than in neuroendocrine carcinoma $(P<0.001)$. After anti-cancer treatment, TROP2 expression was observed in $87 \%(139 / 160)$ of patients. The distribution of TROP2 expression in post-treatment samples was analogous to that in pre-treatment samples when compared using the Wilcoxon signed-rank test $(P=$ 0.509). However, an increase in TROP2 expression was seen in $19(12 \%)$, and a decrease in $20(13 \%)$ patients. Patients treated with targeted therapy showed significantly higher changes in TROP2 expression $(P=0.019)$ and thoracic radiotherapy was more likely to increase TROP2 expression than chemotherapy alone.

Conclusion: TROP2 was expressed in most lung cancer specimens before and after anti-cancer treatments. Additionally, some anti-cancer treatments might alter the TROP2 expression. These results may provide a strong rationale for TROP2-directed therapy against advanced lung cancer.

\section{Introduction}

Trophoblast cell-surface antigen 2 (TROP2), also known as tumor-associated calcium signal transducer 2 (TACSTD2) or epithelial glycoprotein-1 (EGP-1), is a single-pass transmembrane glycoprotein that is expressed on the surface of trophoblast cell and many malignant tumor cells (Cubas et al. 2009; Fornaro et al. 1995; Lipinski et al. 1981; Ripani et al. 1998; Shvartsur and Bonavida 2015; Stepan et al. 2011). Although its function has not been fully clarified, previous reports suggest that overexpression of TROP2 has an anti-apoptotic effect and promotes tumor survival by regulating the mitogen-activated protein kinase/extracellular signal-regulated kinase (MAPK/ERK) pathway or phosphoinositide 3-kinase/protein kinase B (PI3K/Akt) pathway (Cubas et al. 2010; Gu et al. 2018; Li et al. 2019). In fact, it is also known that TROP2 expression in tumor cells is correlated with a poor prognosis in patients with solid cancers (Inamura et al. 2017; Jiang et al. 2013; Kobayashi et al. 2010; Ohmachi et al. 2006; Wu et al. 2013; Xu et al. 2017; Zeng et al. 2016; Zhao et al. 2018). Recently, antibody-drug conjugates (ADCs) targeting TROP2, such as sacituzumab govitecan (IMMU-132) and DS-1062, have been developed. Sacituzumab govitecan is a TROP2-directed ADC that contains SN-38, which is a type I topoisomerase inhibitor that causes 
double-stranded DNA breaks, eventually leading to apoptosis. Previous studies have shown that sacituzumab govitecan has been effective for triple-negative breast cancer, metastatic small cell lung cancer, and metastatic non-small cell lung cancer (NSCLC) that are resistant to other cytotoxic chemotherapy (Bardia et al. 2017; Bardia et al. 2019; Gray et al. 2017; Heist et al. 2017). DS-1062 is also a TROP2-directed ADC that contains a derivative of exatecan (DX-8951), which is a novel type I topoisomerase inhibitor, and has demonstrated clinical activity in advanced NSCLC (Sands et al. 2019). Currently, clinical trials are ongoing to evaluate the safety and efficacy of DS-1062 in advanced NSCLC patients.

According to previous reports, TROP2 expression has been associated with various clinicopathological features and prognosis, in lung cancer patients (Inamura et al. 2017; Jiang et al. 2013; Kobayashi et al. 2010; Pak et al. 2012). However, information on TROP2 expression in advanced lung cancer is insufficient because previous publications have mainly targeted surgical cases, such as early stage lung cancer. Therefore, it is necessary to investigate the prevalence of TROP2 expression in lung cancer tumor cells in a cohort comprising advanced lung cancer patients. In addition, it is still unclear whether TROP2 expression is altered after therapeutic intervention. In this study, we aimed to assess the prevalence of TROP2 expression in lung cancer specimens, before and after administering various anti-cancer treatments, in an advanced lung cancer population.

\section{Materials And Methods}

\section{Patients}

Retrospectively, we screened 169 consecutive patients from the medical records to identify those who were histologically diagnosed with lung cancer and from whom tumor specimens were obtained after treatment, at the Shizuoka Cancer Center between July 2003 and June 2017. This study was approved by the Institutional Review Board of Shizuoka Cancer Center (Approval No. T29-17-29-1; July 27, 2017).

This study included patients with formalin-fixed paraffin-embedded tumor tissue samples sufficient to evaluate TROP2 expression. Pleural fluid cell blocks were evaluated as well as biopsy or surgical tissue samples. If a patient had three or more samples, two samples were selected: one taken at diagnosis and the other obtained after anti-cancer treatment.

Patients who had received one or more anti-cancer treatments, including cytotoxic chemotherapy, epidermal growth factor receptor-tyrosine kinase inhibitor (EGFR-TKI), anaplastic lymphoma kinase (ALK) inhibitor, and curative thoracic radiotherapy, were eligible. Patients who only received surgical resection or palliative radiotherapy were excluded from this study. We evaluated the changes in TROP2 expression before and after anti-cancer therapeutic intervention. Overall survival (OS) was defined as the time from histological diagnosis of lung cancer to the date of death or last contact.

\section{Immunohistochemical assessment}


Patients' formalin-fixed paraffin-embedded samples were sectioned at a thickness of $3 \mu \mathrm{m}$, mounted onto glass slides, and incubated with an anti-rabbit monoclonal antibody against TROP2 (clone: SP295, Daiichi Sankyo Co., Ltd., Tokyo, Japan; diluted 1:72 and clone: SP295, Spring Bioscience Co., California, USA; diluted 1:50). All of the slides were processed on the Autostainer Bond-III platform (Leica Biosystems) and visualized with a Leica Bond Polymer Refine Detection kit (DS9800, Bond Polymer Refine Detection Leica). The sections were incubated at a pH of 9 for $20 \mathrm{~min}$ at $100^{\circ} \mathrm{C}$. After washing in a wash buffer, the slides were incubated for $30 \mathrm{~min}$ at room temperature with the primary antibody. The nuclei were lightly counter-stained with Mayer's hematoxylin. Positive and negative staining controls were provided by Daiichi Sankyo Co., Ltd., Tokyo, Japan.

A blinded histopathological evaluation of TROP2 expression on the membrane of tumor cells was interpreted by two pathologists (Takuya Kawata and Takashi Sugino), with no prior clinical information. Tumor cells with stained cell membranes of the samples were evaluated, and the immunohistochemistry (IHC) scores were graded from 0 to 3 based on IHC human epidermal growth factor receptor 2 (HER2) testing for gastric cancer, as recommended in the American Society of Clinical Oncology/College of American Pathologists Clinical Practice guideline (Bartley et al. 2017). In this study, grade $\geq 2$ was considered to be positive for TROP2 expression. In case of a discrepancy, the pathologists reviewed their assessments and established a consensus. We defined a difference in the IHC score before and after anticancer treatment as the change in TROP2 expression. Additionally, the proportions of changes in IHC scores were evaluated for each anti-cancer treatment.

\section{Statistical methods}

To analyze the correlations between TROP2 expression and patient characteristics, we used Fisher's exact test for categorical variables. The distribution of TROP2 expression between pre-treatment samples and post-treatment samples was compared using the Wilcoxon signed-rank test. Furthermore, we evaluated OS using the Kaplan-Meier method and compared it among lung adenocarcinoma patients diagnosed as stage IV using the log-rank test. Because this study population included various histological types and stages, we selected and analyzed the most predominant population, that is, stage IV adenocarcinoma patients. $P$ values of less than two-sided 0.05 were considered to be statistically significant. All analyses were implemented by JMP 10 for Windows statistical software (SAS Institute Japan Inc., Tokyo, Japan).

\section{Results}

\section{Patient characteristics}

Among the 169 patients who were screened, 9 patients were excluded because the cancer cells in their specimen were not adequate for evaluation; thus, 160 patients were included in this study. The patient characteristics are listed in Table 1. The median patient age at diagnosis was 65 years (range, 39-89 years). Of the 160 patients, 92 (58\%) were male, 49 (31\%) had never smoked, 130 patients ( $81 \%)$ were diagnosed with adenocarcinoma, $19(12 \%)$ with squamous cell carcinoma, and $8(5 \%)$ with

neuroendocrine carcinoma. Fifty patients (31\%) were diagnosed as having stage I or II lung cancer, and 
stage III or IV lung cancer was observed in $110(69 \%)$ patients. Among the 130 adenocarcinoma patients, 42 patients had tumors with deletions in EGFR exon 19, 30 carried the L858R mutation in EGFR exon 21, 3 carried the G719X mutation in EGFR exon 18, and 1 carried an insertion mutation in EGFR exon 20. Among the 45 patients with EGFR wild type adenocarcinoma, 7 had $A L K$-positive lung cancer. Genetic alterations were unknown in 9 patients.

\section{Samples and anti-cancer treatments}

The breakdown of pre-treatment samples was as follows: 95 (59\%) biopsy samples, 58 (36\%) surgically resected specimens, and 7 (5\%) pleural fluid cell blocks. Post-treatment samples included 104 (65\%) biopsy samples, 30 (19\%) pleural fluid cell blocks, 21 (13\%) surgically resected specimens, and $5(3 \%)$ autopsy samples. Pleural fluid cell blocks were more frequently seen in post-treatment samples compared to pre-treatment samples. Table 2 shows the anti-cancer treatments that patients received before their post-treatment samples were obtained. In their clinical course, $123(77 \%)$ patients received cytotoxic chemotherapy, including postoperative adjuvant chemotherapy, and 67 (42\%) received targeted therapy, including EGFR-TKI and ALK-TKI. Thirty-one (19\%) patients received radiation therapy for the primary lesion. However, only $5(3 \%)$ patients were treated with immune checkpoint inhibitors as most patients in this cohort were treated before the approval of immune checkpoint inhibitors in Japan.

\section{TROP2 expression in pre-treatment samples}

Figure 1 shows the patterns of TROP2 staining in the cell membrane of tumor cells. Positive TROP2 staining of tumor cells (IHC score 2 or 3 ) in pre-treatment samples was observed in 143 (89\%) patients. The relationship between patient demographics and TROP2 expression in pre-treatment samples is listed in Table 1. TROP2 was significantly more expressed in adenocarcinoma and squamous cell carcinoma samples than in neuroendocrine carcinoma $(P<0.001)$. While, there was no significant correlation between TROP2 expression and age, sex, smoking status, stage at diagnosis, or the status of EGFR mutation. Similarly, among 110 patients that were diagnosed with stage III or IV lung cancer, positive TROP2 expression was observed in 97 (88\%) patients. However, no significant correlation was observed between TROP2 expression and clinical characteristics except for histology (Table 3).

\section{Changes in TROP2 expression}

Positive TROP2 staining of tumor cells (IHC score 2 or 3 ) in post-treatment samples was observed in 139 (87\%) patients. Among 110 patients with stage III or IV lung cancer, positive TROP2 expression was observed in 94 (85\%) patients. The distribution of TROP2 expression of the post-treatment samples was analogous to those in pre-treatment samples when compared using the Wilcoxon signed-rank test $(P=$ 0.509). However, an increase in TROP2 expression was seen in $19(12 \%)$ patients, while a decrease was observed in 20 (13\%) other patients (Table 4). In adenocarcinoma patients, IHC scores of TROP2 expression were changed by approximately $30 \%$. Among them, a positive or negative conversion of TROP2 expression was observed in 11 (8\%) patients, respectively. 
Regarding the evaluation of anti-cancer treatment intervention, 12 (10\%) of 123 patients treated with cytotoxic chemotherapy showed an increase in TROP2 expression, while a decrease was seen in 15 (12\%) patients (Figure 2). Six (19\%) of 31 patients treated with thoracic radiotherapy showed an increase in TROP2 expression, while a decrease in the same was not observed. Among 67 patients treated with targeted therapy, consisting of EGFR-TKI or ALK-TKI, 10 (15\%) patients showed an increase in TROP2 expression, and 14 (21\%) showed decreased TROP2 expression. Of these patients that were treated with targeted therapy, positive or negative conversion in TROP2 expression occurred in $6(9 \%)$ patients. Of 63 NSCLC patients with EGFR mutations who were treated with EGFR-TKI, an increase in TROP2 expression was observed $9(14 \%)$ patients, while a decrease was observed in 14 (22\%). While comparing each anticancer treatment, patients treated with targeted therapy alone showed a significant change in TROP2 expression compared to those treated with chemotherapy alone $(P=0.019)$. Similarly, patients treated with thoracic radiotherapy were more likely to have an increased TROP2 expression than those undergoing chemotherapy alone $(P=0.063)$.

\section{Survival analysis in stage IV adenocarcinoma patients}

The median follow-up time from diagnosis to censored case was 50.2 months in 71 patients with stage IV adenocarcinoma. There was no significant difference in OS between patients with high TROP2 expressing tumors (IHC score 3 ) and those without high TROP2 expressing tumors (IHC score $0,1,2$ ) (median 38.1 months versus 46.2 months; $P=0.266$; Fig. $3 A$ ). Similarly, no significant difference in OS was observed in patients with stage IV EGFR-mutated adenocarcinoma, who harbored high TROP2 expression and underwent EGFR-TKI treatment, when compared to those without high TROP2 expression (median 43.4 months versus 50.3 months, $P=0.329$; Fig. 3B).

\section{Discussion}

In our study, of the 160 NSCLC patients, TROP2 expression was observed in $89 \%$ of pre-treatment samples. Furthermore, there was no significant change in the prevalence of TROP2 expression in tumor cells in pre- and post-treatment samples $(P=0.509)$. However, while assessing individual patients, changes were observed in 39 (24\%) patients receiving anti-cancer treatments during their clinical course. To the best of our knowledge, no reports that evaluated the changes in TROP2 expression among lung cancer patients, including advanced-stage lung cancer, have been published. Our analysis contributes novel data on TROP2 expression in patients with advanced lung cancer, adding value to the existing body of TROP2 research.

In previous reports, TROP2 expression in lung cancer has been described using archived surgically resected specimens. Inamura $\mathrm{K}$, et al. reported the association of TROP2 expression in tumor cells by investigating the clinicopathological or molecular characteristics and prognosis in lung cancer patients. Their results depicted a high TROP 2 expression in $64 \%$ of adenocarcinoma, $75 \%$ of squamous cell carcinoma, and $18 \%$ of high grade neuroendocrine carcinoma patients using a rabbit monoclonal antiTROP2 antibody (Clone 1E5-1E2) (Inamura et al. 2017). Pak MG et al. also reported that patients with 
lung squamous cell carcinoma showed significantly higher TROP2 expression in tumor cells, compared to patients with lung adenocarcinoma ( $64 \%$ vs $23 \%, P<0.01)$ (Pak et al. 2012). Although the definitions of positive TROP2 expression were different, these results are consistent with our findings.

TROP2-directed ADCs have been reported to have potential as new therapeutic agents for lung and breast cancers (Gray et al. 2017; Heist et al. 2017). In a recent phase I study of a TROP2-directed ADC (DS-1062), drug clinical activity was observed irrespective of the levels of TROP2 expression (Lisberg et al. 2020). However, considering the mechanisms of action of $A D C$ treatments, there is no doubt that the status of TROP2 expression in tumor cells plays an essential role in TROP2-directed ADC treatment. Our study demonstrated that TROP2 was expressed in most lung cancer specimens, before and after various anticancer treatments. This stable high expression rate of TROP2 in advanced lung cancer, during the clinical course, supports the rationale for TROP2-directed ADC treatments.

Although we observed that the frequency of TROP2 expression did not significantly differ after treatment, changes in IHC score were observed in approximately $20-30 \%$ of patients. Considering the results of our research, we hypothesize that anti-cancer treatments, including chemotherapy and radiotherapy, may change TROP2 expression in some lung cancer patients. Concerning the pathophysiology of changes in TROP2 expression, Zhao P et al. reported that tumor necrosis factor-alpha (TNF-a) can promote cancer cell migration and invasion by upregulating TROP2 expression (Zhao and Zhang 2018). Induction or reduction of TNF-a by therapeutic intervention may result in an altered tumor microenvironment, and consequently, change the TROP2 expression. In our study, patients that were only treated with targeted therapy showed a significant change in TROP2 expression than those who underwent chemotherapy alone $(P=0.019)$. Additionally, patients treated with radiotherapy were more likely to show an increase in TROP2 expression than those treated with chemotherapy alone $(P=0.063)$. Thus, targeted therapy and radiotherapy may enhance TNF-a production, as opposed to chemotherapy, and that may lead to increased TROP2 expression. However, in this retrospective study, we used archived tumor tissues, and therefore, we could not evaluate the changes in TNF-a. Since the pathophysiology of TROP2 expression is not fully interpreted, further research is needed to investigate whether TROP2 expression may change spontaneously, during the clinical course, regardless of intervention by anti-cancer treatments.

Regarding survival analysis, although there was no significant difference, we observed a potential for worse survival outcomes in patients with high TROP2-expressing lung adenocarcinoma, including EGFRmutated adenocarcinoma. This is consistent with previously reported findings in lung cancer patients (Inamura et al. 2017; Jiang et al. 2013; Kobayashi et al. 2010). Mito R et al. reported that TROP2 expression is a prognostic factor in adenocarcinoma patients without EGFR mutation as well as those with a high histological grade (Mito et al. 2020). They also reported an absence of correlation between TROP2 expression and prognosis for adenocarcinoma patients harboring EGFR mutation. Since more than $70 \%$ of the patients were stage 0 or 1 , we assume that there was no correlation between TROP2 expression and survival in adenocarcinoma patients harboring EGFR mutation in their cohort (KaplanMeier OS curves had less than $20 \%$ of death events during the observation period) (Mito et al. 2020). Previous systematic reviews, involving other solid cancers, have also shown that TROP2 expression is 
associated with having a poor prognosis (Xu et al. 2017; Zeng et al. 2016). Conversely, Pak et al. suggested that TROP2 expression is associated with favorable OS in lung adenocarcinoma patients (Pak et al. 2012). The cause of such a discrepancy in lung cancer is still unknown and therefore, further studies are required.

Our study had several limitations. First, it was a single-institutional, retrospective study. Second, the patient characteristics were heterogeneous: we included patients in various cancer stages, undergoing various anti-cancer treatments. Third, it may not show an accurate prevalence of TROP2 expression, especially in small biopsy samples due to spatial heterogeneity in TROP2 expression within different regions of the same tumor tissue. To the best of our knowledge, present-day data showing intra-tumoral heterogeneity of TROP2 expression in lung cancer have not been reported. However, given the complexity of tumor microenvironment, it is likely that intra-tumoral heterogeneity of TROP2 expression may exist. Concerning the level of TROP2 expression based on the type of tumor specimens, there was no significant difference between surgically resected specimens and biopsy samples in our study. Therefore, even if intra-tumoral heterogeneity of TROP2 expression does exist, its assessment using biopsy samples would be acceptable. Fourth, as previously described, other molecules, such as TNF- $a$, were not evaluated, which may be involved in the pathophysiology of altered TROP2 expression.

In conclusion, TROP2 was expressed in most lung cancer specimens of both the early and advanced stages, particularly in those of adenocarcinoma and squamous cell carcinoma. Although there was no significant change in the prevalence of TROP2 expression in pre- and post-treatment samples, anti-cancer treatments, especially targeted therapy and radiotherapy, might alter TROP2 expression in advanced lung cancer patients. These results support the rationale for TROP2-directed therapy, which could yield a therapeutic effect against advanced lung cancer in different lines of treatment after various therapeutic interventions.

\section{Declarations}

\section{Funding:}

This study was supported by a contract research fund from Daiichi Sankyo Co., Ltd., Tokyo, Japan.

\section{Conflicts of interest/Competing interests:}

The authors declare no conflict of interest directly relevant or directly related to the content of this article.

\section{Availability of data and material:}

The remaining data that support the findings of this study are available from the corresponding author upon reasonable request. The data are not publicly available due to privacy or ethical restrictions.

\section{Code availability:}


Not applicable

\section{Authors' contributions:}

Shota Omori was involved in study concept and design; acquisition, analysis, or interpretation of data; drafting of the manuscript; full access to all data in the study and responsible for the integrity of the data and accuracy of the data analysis. Koji Muramatsu, Takuya Kawata, and Takashi Sugino were involved in pathological support. Eriko Miyawaki, Taichi Miyawaki, Nobuaki Mamesaya, Takahisa Kawamura, Haruki Kobayashi, Kazuhisa Nakashima, Kazushige Wakuda, Akira Ono, Tateaki Naito, Haruyasu Murakami were involved in material support. Hirotsugu Kenmotsu was involved in administrative, technical or material support. Toshiaki Takahashi was involved in study supervision. All authors read and approved the final manuscript.

\section{Ethics approval:}

This study was approved by the Institutional Review Board of the Shizuoka Cancer Center, Japan (Approval No. T29-17-29-1; July 27, 2017).

\section{Consent to participate:}

An opt-out method was used on the basis of the Ethical Guidelines for Epidemiological Research in Japan.

\section{Consent for publication:}

Not applicable

\section{Acknowledgments:}

We thank all the patients who participated in this study and their families. We also thank all the clinical pathology technicians who supported this study.

\section{References}

1. Bardia A et al. (2017) Efficacy and Safety of Anti-Trop-2 Antibody Drug Conjugate Sacituzumab Govitecan (IMMU-132) in Heavily Pretreated Patients With Metastatic Triple-Negative Breast Cancer. J Clin Oncol 35:2141-2148. https://doi.org/10.1200/JC0.2016.70.8297

2. Bardia A et al. (2019) Sacituzumab Govitecan-hziy in Refractory Metastatic Triple-Negative Breast Cancer. N Engl J Med 380:741-751. https://doi.org/10.1056/NEJMoa1814213

3. Bartley AN et al. (2017) HER2 Testing and Clinical Decision Making in Gastroesophageal Adenocarcinoma: Guideline From the College of American Pathologists, American Society for Clinical Pathology, and the American Society of Clinical Oncology. J Clin Oncol 35:446-464. https://doi.org/10.1200/JCO.2016.69.4836 
4. Cubas R, Li M, Chen C, Yao Q (2009) Trop2: a possible therapeutic target for late stage epithelial carcinomas. Biochim Biophys Acta 1796:309-314. https://doi.org/10.1016/j.bbcan.2009.08.001

5. Cubas R, Zhang S, Li M, Chen C, Yao Q (2010) Trop2 expression contributes to tumor pathogenesis by activating the ERK MAPK pathway. Mol Cancer 9:253. https://doi.org/10.1186/1476-4598-9-253

6. Fornaro M, Dell'Arciprete R, Stella M, Bucci C, Nutini M, Capri MG, Alberti S (1995) Cloning of the gene encoding Trop-2, a cell-surface glycoprotein expressed by human carcinomas. Int J Cancer 62:610618. https://doi.org/10.1002/ijc.2910620520

7. Gray JE et al. (2017) Therapy of Small Cell Lung Cancer (SCLC) with a Topoisomerase-l-inhibiting Antibody-Drug Conjugate (ADC) Targeting Trop-2, Sacituzumab Govitecan. Clin Cancer Res 23:57115719. https://doi.org/10.1158/1078-0432.CCR-17-0933

8. Gu QZ, Nijiati A, Gao X, Tao KL, Li CD, Fan XP, Tian Z (2018) TROP2 promotes cell proliferation and migration in osteosarcoma through PI3K/AKT signaling. Mol Med Rep 18:1782-1788. https://doi.org/10.3892/mmr.2018.9083

9. Heist RS et al. (2017) Therapy of Advanced Non-Small-Cell Lung Cancer With an SN-38-Anti-Trop-2 Drug Conjugate, Sacituzumab Govitecan. J Clin Oncol 35:2790-2797. https://doi.org/10.1200/JC0.2016.72.1894

10. Inamura $\mathrm{K}$ et al. (2017) Association of tumor TROP2 expression with prognosis varies among lung cancer subtypes. Oncotarget 8:28725-28735. https://doi.org/10.18632/oncotarget.15647

11. Jiang A, Gao X, Zhang D, Zhang L, Lu H (2013) Expression and clinical significance of the Trop-2 gene in advanced non-small cell lung carcinoma. Oncol Lett 6:375-380.

https://doi.org/10.3892/ol.2013.1368

12. Kobayashi $\mathrm{H}$ et al. (2010) Expression of the GA733 gene family and its relationship to prognosis in pulmonary adenocarcinoma. Virchows Arch 457:69-76. https://doi.org/10.1007/s00428-010-0930-8

13. Li X et al. (2019) Correction: TROP2 promotes proliferation, migration and metastasis of gallbladder cancer cells by regulating PI3K/AKT pathway and inducing EMT. Oncotarget 10:6540. https://doi.org/10.18632/oncotarget.27299

14. Lipinski M, Parks DR, Rouse RV, Herzenberg LA (1981) Human trophoblast cell-surface antigens defined by monoclonal antibodies. Proc Natl Acad Sci U S A 78:5147-5150. https://doi.org/10.1073/pnas.78.8.5147

15. Lisberg AE et al. (2020) Dose escalation and expansion from the phase I study of DS-1062, a trophoblast cell-surface antigen 2 (TROP2) antibody drug conjugate (ADC), in patients (pts) with advanced non-small cell lung cancer (NSCLC). Journal of Clinical Oncology 38:9619-9619. https://doi.org/10.1200/JC0.2020.38.15_suppl.9619

16. Mito R et al. (2020) Clinical impact of TROP2 in non-small lung cancers and its correlation with abnormal p53 nuclear accumulation. Pathol Int. https://doi.org/10.1111/pin.12911

17. Ohmachi T, Tanaka F, Mimori K, Inoue H, Yanaga K, Mori M (2006) Clinical significance of TROP2 expression in colorectal cancer. Clin Cancer Res 12:3057-3063. https://doi.org/10.1158/10780432.CCR-05-1961 
18. Pak MG, Shin DH, Lee CH, Lee MK (2012) Significance of EpCAM and TROP2 expression in nonsmall cell lung cancer. World J Surg Oncol 10:53. https://doi.org/10.1186/1477-7819-10-53

19. Ripani E, Sacchetti A, Corda D, Alberti S (1998) Human Trop-2 is a tumor-associated calcium signal transducer. Int J Cancer 76:671-676. https://doi.org/10.1002/(sici)10970215(19980529)76:5<671::aid-ijc10>3.0.co;2-7

20. Sands JM et al. (2019) First-in-human phase 1 study of DS-1062a in patients with advanced solid tumors. Journal of Clinical Oncology 37:9051-9051. https://doi.org/10.1200/JC0.2019.37.15_suppl.9051

21. Shvartsur A, Bonavida B (2015) Trop2 and its overexpression in cancers: regulation and clinical/therapeutic implications. Genes Cancer 6:84-105. https://doi.org/10.18632/genesandcancer.40

22. Stepan LP, Trueblood ES, Hale K, Babcook J, Borges L, Sutherland CL (2011) Expression of Trop2 cell surface glycoprotein in normal and tumor tissues: potential implications as a cancer therapeutic target. J Histochem Cytochem 59:701-710. https://doi.org/10.1369/0022155411410430

23. Wu H et al. (2013) Potential therapeutic target and independent prognostic marker of TROP2 in laryngeal squamous cell carcinoma. Head Neck 35:1373-1378. https://doi.org/10.1002/hed.23138

24. Xu P et al. (2017) Prognostic role and clinical significance of trophoblast cell surface antigen 2 in various carcinomas. Cancer Manag Res 9:821-837. https://doi.org/10.2147/CMAR.S147033

25. Zeng P, Chen MB, Zhou LN, Tang M, Liu CY, Lu PH (2016) Impact of TROP2 expression on prognosis in solid tumors: A Systematic Review and Meta-analysis. Sci Rep 6:33658. https://doi.org/10.1038/srep33658

26. Zhao P, Zhang Z (2018) TNF-alpha promotes colon cancer cell migration and invasion by upregulating TROP-2. Oncol Lett 15:3820-3827. https://doi.org/10.3892/ol.2018.7735

27. Zhao W et al. (2018) Trop2 is a potential biomarker for the promotion of EMT in human breast cancer. Oncol Rep 40:759-766. https://doi.org/10.3892/or.2018.6496

\section{Tables}


Table 1

Patients' characteristics and pre-treatment TROP2 expression

\begin{tabular}{|c|c|c|c|c|}
\hline & \multirow{2}{*}{$\begin{array}{l}\text { No. of } \\
\text { Patients }\end{array}$} & \multicolumn{2}{|c|}{ TROP2 expression } & \multirow[t]{2}{*}{ P-value } \\
\hline & & $\begin{array}{l}\text { Negative } \\
\text { (IHC score } \\
0,1) \\
n=17\end{array}$ & $\begin{array}{l}\text { Positive } \\
\text { (IHC score } \\
2,3 \text { ) } \\
n=143\end{array}$ & \\
\hline \multicolumn{5}{|l|}{ Age at diagnosis (years) } \\
\hline$\leq 65$ & 82 & 10 & 72 & 0.611 \\
\hline$>65$ & 78 & 7 & 71 & \\
\hline \multicolumn{5}{|l|}{ Sex } \\
\hline Male & 92 & 13 & 79 & 0.121 \\
\hline Female & 68 & 4 & 64 & \\
\hline \multicolumn{5}{|l|}{ Smoking status } \\
\hline Previous/current & 111 & 14 & 97 & 0.275 \\
\hline Never smoker & 49 & 3 & 46 & \\
\hline \multicolumn{5}{|l|}{ Histology } \\
\hline Adenocarcinoma & 130 & 10 & 120 & $<0.001$ \\
\hline Squamous carcinoma & 19 & 0 & 19 & $\begin{array}{l}(* \\
<0.001)\end{array}$ \\
\hline Neuroendocrine carcinoma & 8 & 6 & 2 & \\
\hline Others & 3 & 1 & 2 & \\
\hline \multicolumn{5}{|l|}{ Stage at diagnosis } \\
\hline I, II & 50 & 4 & 46 & 0.586 \\
\hline III, IV & 110 & 13 & 97 & \\
\hline $\begin{array}{l}\text { EGFR mutation status in Adenocarcinoma } \\
\text { patients }\end{array}$ & $N=130$ & $N=10$ & $N=120$ & \\
\hline Mutant & 76 & 5 & 71 & 0.563 \\
\hline Wild type & 45 & 5 & 40 & \\
\hline Unknown & 9 & 0 & 9 & \\
\hline
\end{tabular}




\begin{tabular}{|c|c|c|}
\hline \multirow{5}{*}{$\begin{array}{l}\text { No. of } \\
\text { Patients }\end{array}$} & \multicolumn{2}{|c|}{ TROP2 expression } \\
\hline & Negative & Positive \\
\hline & (IHC score & (IHC score \\
\hline & $0,1)$ & $2,3)$ \\
\hline & $n=17$ & $n=143$ \\
\hline
\end{tabular}

*: Fisher's test for combined Adenocarcinoma and Squamous cell carcinoma vs. Neuroendocrine carcinoma

Abbreviation: TROP2, trophoblast cell-surface antigen 2; IHC, immunohistochemical; EGFR, epidermal growth factor receptor

Table 2

The anti-cancer treatments that patients received before posttreatment samples were obtained

\begin{tabular}{|ll|}
\hline & No. of patients \\
\hline $\begin{array}{l}\text { Cytotoxic chemotherapy } \\
\text { (including adjuvant chemotherapy) }\end{array}$ & 62 \\
\hline Cytotoxic chemotherapy and Targeted therapy* & 42 \\
\hline Targeted therapy & 25 \\
\hline Chemoradiotherapy & 19 \\
\hline Radiotherapy & 12 \\
\hline $\begin{array}{l}\text { *: Targeted Therapy; EGFR-TKI or ALK-TKI } \\
\text { Abbreviation: EGFR, epidermal growth factor receptor; } \\
\text { ALK, anaplastic lymphoma kinase; TKI, tyrosine kinase inhibitor }\end{array}$ \\
\hline
\end{tabular}


Table 3

Patients' characteristics and pre-treatment TROP2 expression (stage III/IV)

\begin{tabular}{|c|c|c|c|c|}
\hline & \multirow{2}{*}{$\begin{array}{l}\text { No. of } \\
\text { Patients }\end{array}$} & \multicolumn{2}{|c|}{ TROP2 expression } & \multirow[t]{2}{*}{ P-value } \\
\hline & & $\begin{array}{l}\text { Negative } \\
\text { (IHC score } \\
0,1) \\
n=13\end{array}$ & $\begin{array}{l}\text { Positive } \\
\text { (IHC score } \\
2,3 \text { ) } \\
n=97\end{array}$ & \\
\hline \multicolumn{5}{|l|}{ Age at diagnosis (years) } \\
\hline$\leq 65$ & 57 & 8 & 49 & 0.455 \\
\hline$>65$ & 53 & 5 & 48 & \\
\hline \multicolumn{5}{|l|}{ Sex } \\
\hline Male & 56 & 9 & 47 & 0.159 \\
\hline Female & 54 & 4 & 50 & \\
\hline \multicolumn{5}{|l|}{ Smoking status } \\
\hline Previous/current & 73 & 10 & 63 & 0.390 \\
\hline Never smoker & 37 & 3 & 34 & \\
\hline \multicolumn{5}{|l|}{ Histology } \\
\hline Adenocarcinoma & 96 & 9 & 87 & $<0.001$ \\
\hline Squamous carcinoma & 9 & 0 & 9 & $\begin{array}{l}(* \\
<0.001)\end{array}$ \\
\hline Neuroendocrine carcinoma & 5 & 4 & 1 & \\
\hline \multicolumn{5}{|l|}{ Others } \\
\hline \multicolumn{5}{|l|}{ Stage at diagnosis } \\
\hline I, II & 0 & 0 & 0 & - \\
\hline III, IV & 110 & 13 & 97 & \\
\hline $\begin{array}{l}\text { EGFR mutation status in Adenocarcinoma } \\
\text { patients }\end{array}$ & $N=96$ & $N=9$ & $N=87$ & \\
\hline Mutant & 63 & 5 & 58 & 0.379 \\
\hline Wild type & 29 & 4 & 25 & \\
\hline Unknown & 4 & 0 & 4 & \\
\hline
\end{tabular}




\begin{tabular}{lll} 
No. of & \multicolumn{2}{l}{ TROP2 expression } \\
\cline { 2 - 3 } Patients & Negative & Positive \\
& (IHC score & (IHC score \\
& $0,1)$ & $2,3)$ \\
& $n=13$ & $n=97$
\end{tabular}

*: Fisher's test for combined Adenocarcinoma and Squamous cell carcinoma vs. Neuroendocrine carcinoma

Abbreviation: TROP2, trophoblast cell-surface antigen 2; IHC, immunohistochemical; EGFR, epidermal growth factor receptor

Table 4

TROP2 expression before and after treatment for each lung cancer subtype

\begin{tabular}{|c|c|c|c|c|c|c|c|c|c|c|c|}
\hline & \multicolumn{4}{|c|}{$\begin{array}{l}\text { TROP2 expression } \\
\text { before treatment }\end{array}$} & \multicolumn{4}{|c|}{$\begin{array}{l}\text { TROP2 expression } \\
\text { after treatment }\end{array}$} & \multicolumn{3}{|c|}{ Change in TROP2 expression } \\
\hline & \multicolumn{2}{|c|}{$\begin{array}{l}\text { Negative } \\
(0,1)\end{array}$} & \multicolumn{2}{|c|}{$\begin{array}{l}\text { Positive } \\
(2,3)\end{array}$} & \multicolumn{2}{|c|}{$\begin{array}{l}\text { Negative } \\
(0,1)\end{array}$} & \multicolumn{2}{|c|}{$\begin{array}{l}\text { Positive } \\
(2,3)\end{array}$} & \multirow{2}{*}{$\begin{array}{l}\text { Increase } \\
\%\end{array}$} & \multirow{2}{*}{$\begin{array}{l}\begin{array}{l}\text { No } \\
\text { change }\end{array} \\
\%\end{array}$} & \multirow{2}{*}{$\begin{array}{l}\text { Decrease } \\
\%\end{array}$} \\
\hline & $\mathbf{N}$ & $\%$ & $\mathbf{N}$ & $\%$ & $\mathbf{N}$ & $\%$ & $\mathbf{N}$ & $\%$ & & & \\
\hline $\begin{array}{l}\text { Total } \\
(\mathrm{N}= \\
160)\end{array}$ & 17 & $11 \%$ & 143 & $89 \%$ & 21 & $13 \%$ & 139 & $87 \%$ & $12 \%$ & $76 \%$ & $13 \%$ \\
\hline $\begin{array}{l}\mathrm{Ad}(\mathrm{N} \\
=130)\end{array}$ & 10 & $8 \%$ & 120 & $92 \%$ & 14 & $11 \%$ & 116 & $89 \%$ & $14 \%$ & $72 \%$ & $15 \%$ \\
\hline $\begin{array}{l}\text { SqCC } \\
(\mathrm{N}= \\
19)\end{array}$ & 0 & $0 \%$ & 19 & $100 \%$ & 0 & $0 \%$ & 19 & $100 \%$ & $0 \%$ & $100 \%$ & $0 \%$ \\
\hline $\begin{array}{l}\text { NEC } \\
(\mathrm{N}=8)\end{array}$ & 6 & $75 \%$ & 2 & $25 \%$ & 5 & $63 \%$ & 3 & 378 & $13 \%$ & $88 \%$ & $0 \%$ \\
\hline $\begin{array}{l}\text { Others } \\
(\mathrm{N}=3)\end{array}$ & 1 & $33 \%$ & 2 & $67 \%$ & 2 & $67 \%$ & 1 & $33 \%$ & $0 \%$ & $67 \%$ & $33 \%$ \\
\hline
\end{tabular}

\section{Figures}



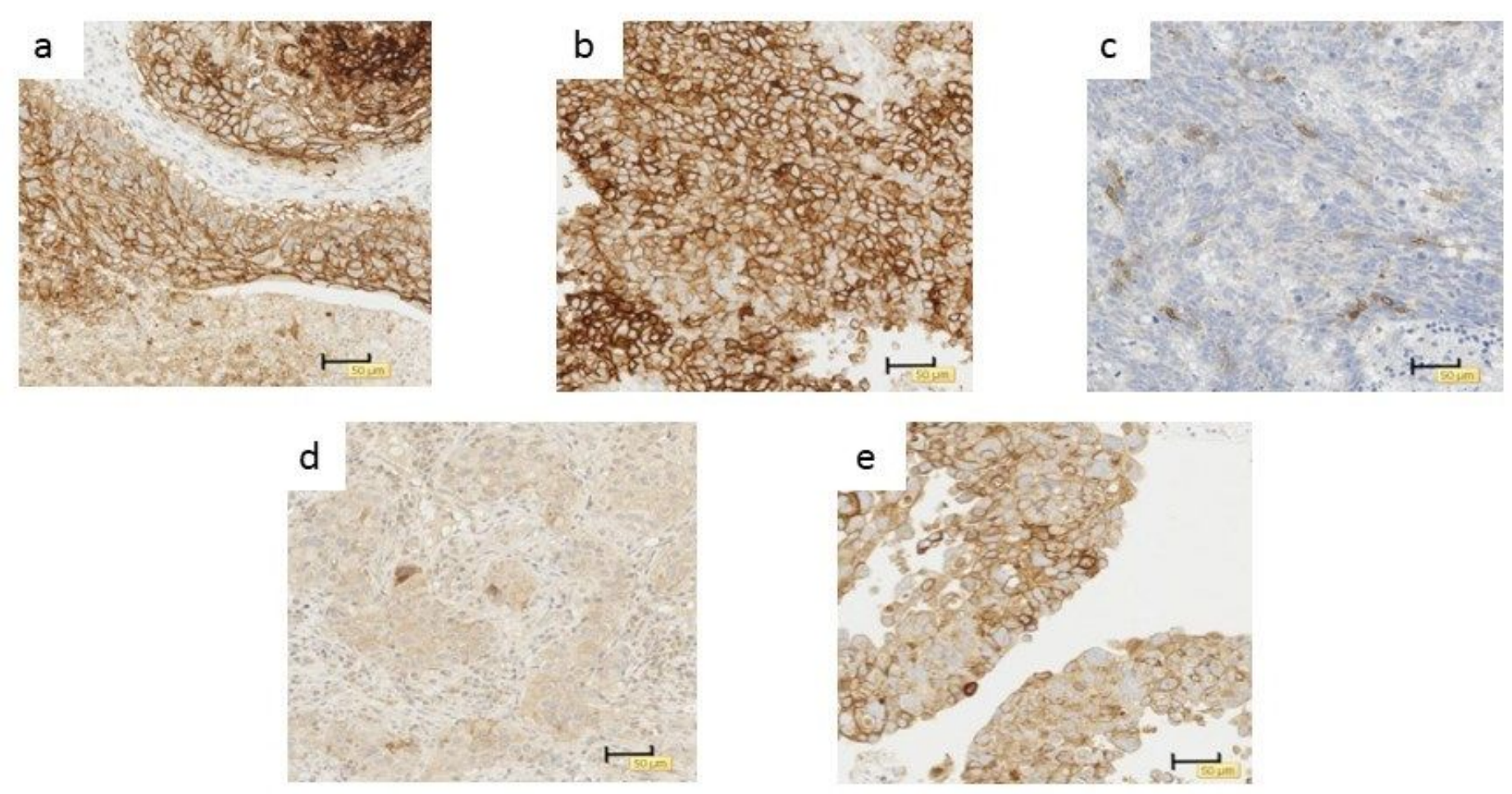

\section{Figure 1}

Immunohistochemical staining of TROP2. TROP2 expression in squamous cell carcinoma (a) and large cell neuroendocrine carcinoma (b) with high TROP2 expression (IHC score 3), small cell carcinoma (c) with negative TROP2 expression (IHC score 0), adenocarcinoma (d) with negative TROP2 expression (IHC score 1) before treatment, and adenocarcinoma (e) with positive TROP2 expression (IHC score 3 ) after treatment. (d) and (e) are the same patients' samples with increasing TROP2 expression after treatment.

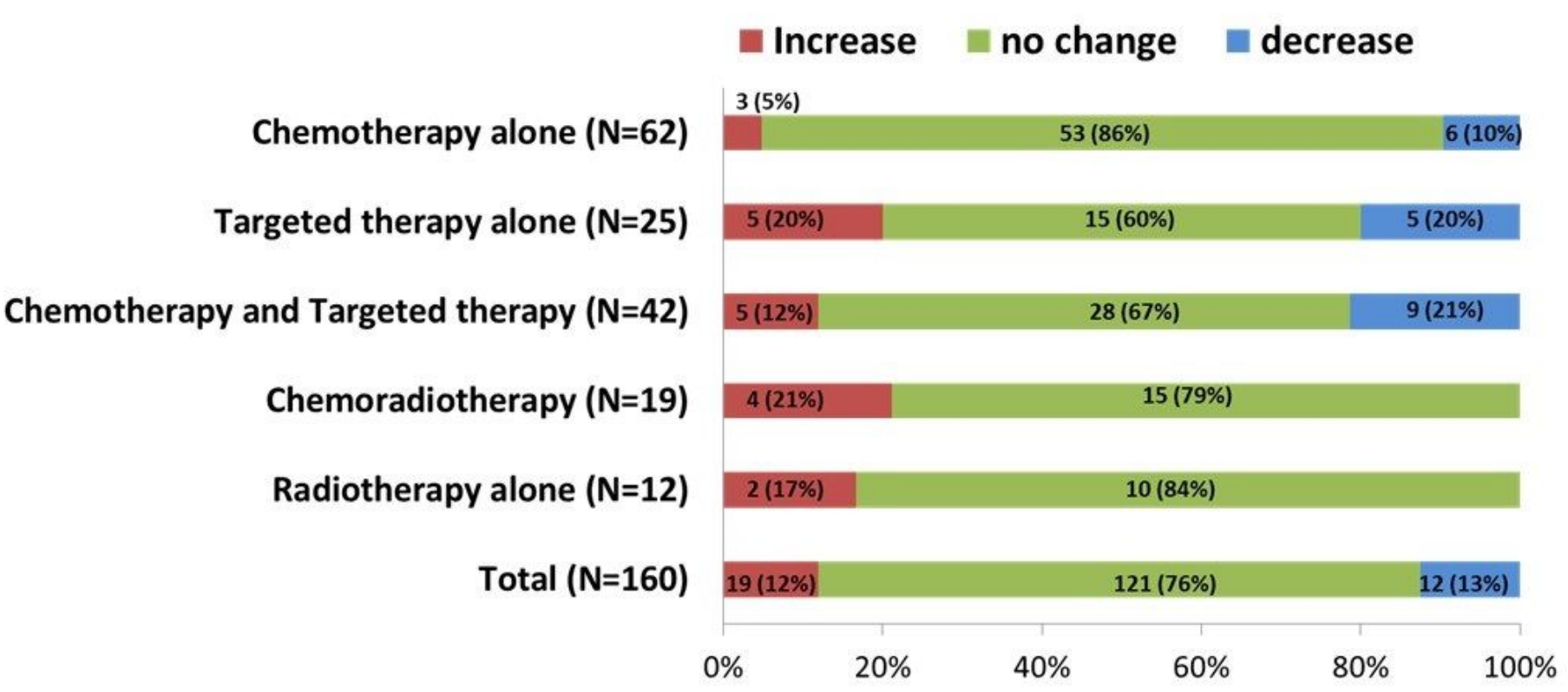


Figure 2

Changes in TROP 2 expression by anti-cancer treatment. Patients treated with targeted therapy alone showed a significant increase in TROP2 expression compared to those treated with chemotherapy alone $(P=0.040)$. Similarly, patients treated with thoracic radiotherapy (chemoradiotheray and radiotherapy alone) were more likely to have an increased TROP2 expression than those undergoing chemotherapy alone $(P=0.063)$.
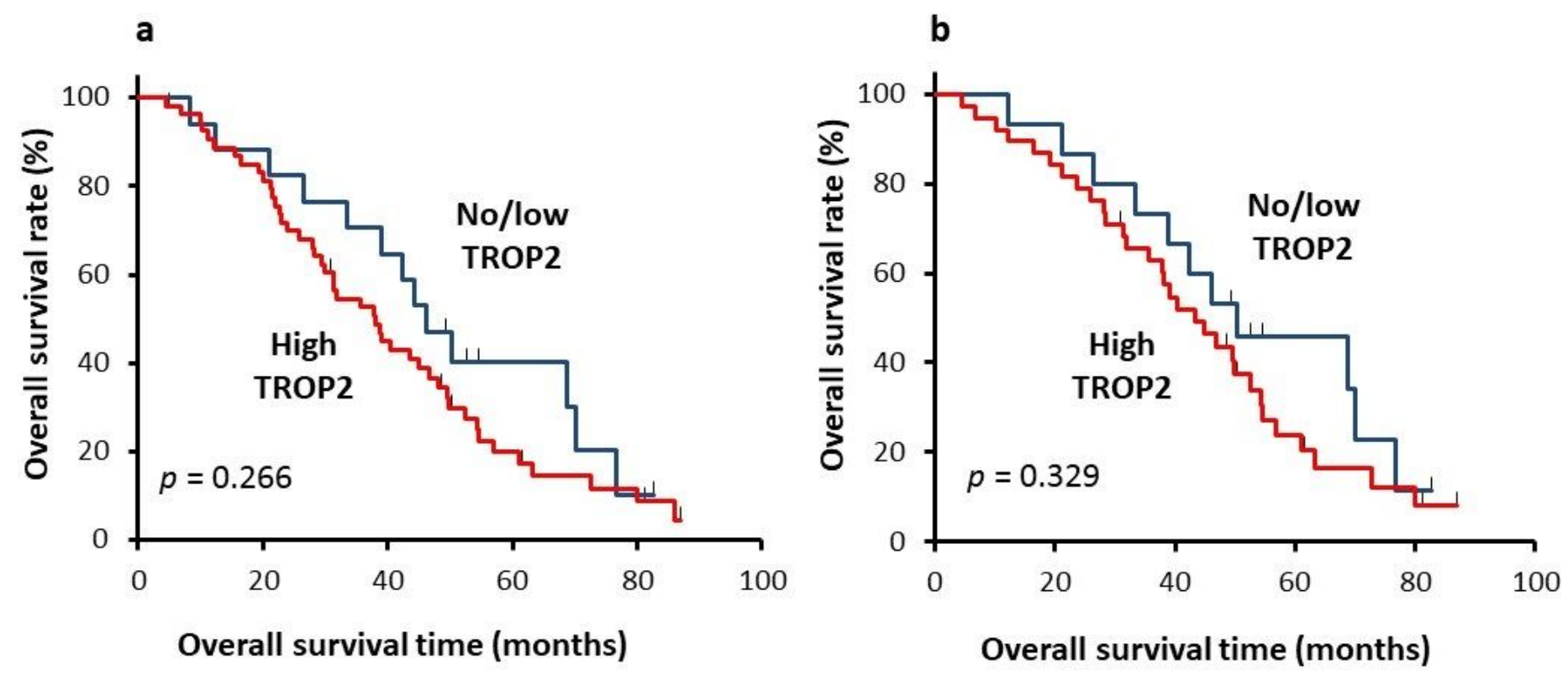

Figure 3

Overall survival according to high TROP2 expression in stage IV adenocarcinoma patients (a), and stage IV EGFR-mutated patients (b). There was no significant difference in overall survival between patients with high TROP2 expressing tumors (IHC score 3 ) and in those without high TROP2 expressing tumors (IHC score $0,1,2$ ) (median 38.1 months versus 46.2 months, $P=0.266$; [a]). Similarly, no significant difference in overall survival was observed in patients with stage IV EGFR-mutated adenocarcinoma, who harbored high TROP2 expression and underwent EGFR-TKI treatment, when compared to those without high TROP2 expression (median 43.4 months versus 50.3 months, $\mathrm{P}=0.329 ;$ [b]). 